\title{
Statistical analysis of the mismatch negativity: To a dilemma, an answer
}

\author{
Maude Beauchemin ${ }^{1,2} \quad$ Louis De Beaumont $^{1}$ \\ ${ }^{1}$ Université de Montréal \\ ${ }^{2}$ Hôpital Ste-Justine
}

\begin{abstract}
This paper offers a new innovative outlook on mismatch negativity (MMN) analysis. Indeed, researchers in this field encounter difficulties when attempting to objectively quantify the MMN component waveform. Advantages taken from already existing amplitude and area under the curve measures were used in order to thwart weaknesses from each individual measure. The present paper can also be used as a guideline that describes each step required in the execution of the proposed technique to MMN analysis.
\end{abstract}

Ce travail suggère une nouvelle approche à l'analyse de la MMN. En effet, certains problèmes sont engendrés par les outils couramment utilisés pour analyser la MMN, notamment l'amplitude et l'aire sous la courbe. La technique suggérée afin de développer une mesure objective de la MMN propose d'utiliser les forces des deux techniques précédemment nommées afin de pallier à leurs faiblesses respectives. Le présent travail se veut également un mode d'emploi quant à la façon d'appliquer les étapes nécessaires à la réalisation de cette nouvelle approche à l'analyse de la MMN.

We first wanted to do a tutorial about the BrainVision Analyser program, which processes raw EEG data both for spontaneous EEG analyses and for evoked potentials. However, its user-friendly workspace designed to allow users to interactively compute complex analysis tasks combined with the already existing comprehensive Vision Analyser User Manual (version 1.05 (c) Brain Products GmbH 1999 - 2004), which contains detailed information on how to design a multi-step analysis, have changed our plans. In fact, we did not want this paper to be a replicate of what had already been made accessible to the public. Instead, we have decided to propose a new perspective on Mismatch Negativity (MMN) analysis. This paper begins with a brief introduction on what the MMN component stands for, its origins and its associated variables of interest. We will then present a dilemma frequently encountered by researchers conducting ERP studies using the MMN component and provide you with what we consider the most appropriate way to resolve the issue.

\section{EEG, ERP and MMN}

The voltage difference between an electrode placed at a position of interest on the scalp and a reference electrode, placed at a relatively neutral position with respect to the neural activity of interest, yield an electroencephalogram (EEG). More specifically, the EEG is a time-varying voltage signal that reflects the activity of many neurons working in concert (Fig. 1).

Figure 1. An EEG trace

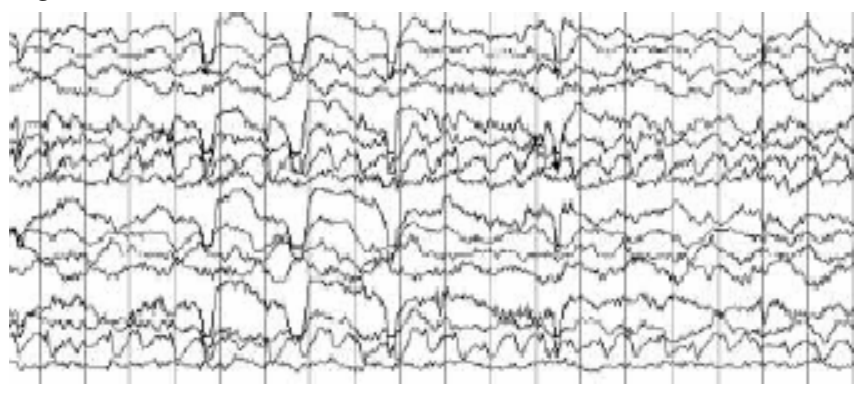


If a stimulus event, such as a sound, is presented, some of the measured neural activity will reflect the processing of that sound event. This activity is termed the event-related potential (ERP). However, on a single trial, the neural activity unrelated to the sound event, which is usually referred to as "noise", typically precludes observation of the ERP waveform of interest. Thus, many trials of the sound event must be administered. The resulting waveforms are afterwards lined up according to the onset of the sound events and then averaged (Fig. 2).

Figure 2. Once averaged, this is what an ERP wave looks like. Note the ERP components, e.g. P1 (positive component peaking at about $100 \mathrm{~ms}$ ).

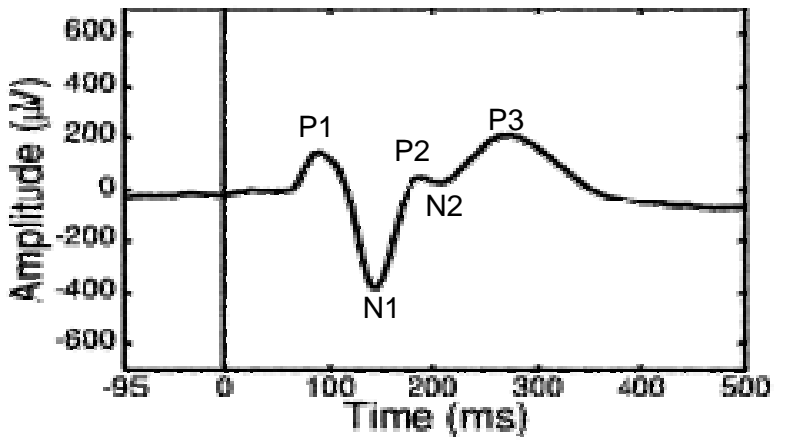

The MMN is a change-specific component of the auditory event-related potentials (ERPs). Indeed, the MMN is a versatile measure that can discriminate the smallest alterations when any one parameter differs between two consecutive stimuli. Auditory oddball paradigms, which involve the presentation of infrequent stimuli embedded among frequent stimuli, have commonly been used to generate the ERP component called MMN (Näätänen et al., 1978; Näätänen \& Alho, 1995). According to Näätänen and Alho's (1995) model, the discrimination of two successive stimuli differing in only one parameter reflects the involvement of two different neural representations. In other words, a frequently presented stimulus forms a neural trace in the echoic sensory memory, which can last up to 8 to10 seconds (Böttcher-Gandor \& Ullsperger, 1992). The sensory input from the deviant stimulus does not fit with the existing neural trace, therefore resulting in a negative deflection, the MMN component. Thus, the MMN is elicited by any discriminable change in some repetitive aspects of auditory stimulation stored in echoic memory. Importantly, the MMN is not elicited by those deviant stimuli when standard stimuli are omitted (Näätänen, 1995), implying that the MMN indexes the discrepancy between the incoming stimulus and the memory representation of the standard stimulus (Cowan et al., 1993). In adults, the maximum amplitude of this negative deflection is obtained over the frontal and central regions of the scalp, suggesting that its primary source is located in the supratemporal auditory cortex (Girard et al., 1990). Moreover, this MMN occurs roughly $200 \mathrm{~ms}$ after stimulus onset and necessitates 200-250 presentations of the deviant stimulus in order to obtain a reliable and consistent MMN waveform component (McGee et al., 1997). The MMN is usually computed as ERPs evoked by a standard stimulus are subtracted from ERPs evoked by the presentation of a deviant stimulus (Fig. 3). The most common variables examined when studying MMN are the amplitude (in $\mu \mathrm{V}$ ), the latency from stimulus onset (in $\mathrm{ms}$ ) and the area under the curve (in $\mu V^{*}$ ms) of the peak of interest.

Figure 3. The MMN wave is a subtraction of the ERP to the standard stimulus from the ERP to the deviant stimulus. Note that in this figure, the polarity is inverted (negative up).
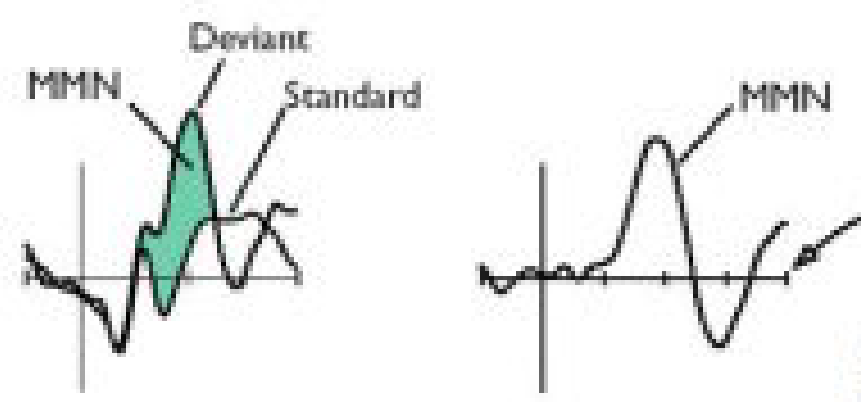

To illustrate the application of the dilemma that will be presented in section 4 , the next section of this paper will describe our latest study using the MMN component.

\section{Presentation of a MMN study}

(Beauchemin, De Beaumont, Turcotte, Arcand, Vannasing, Belin, \& Lassonde, 2005)

The MMN has received substantial scientific attention in the last decades as it is thought to reflect (a) the activation of cerebral mechanisms essential to pre-attentive auditory discrimination and (b) the echoic sensory memory that underlies the latter discrimination process. This growing interest toward the $\mathrm{MMN}$ has originated as considerable efforts have been exerted to disclose an objective measure of primary auditory information processing capacities. The current study sought to determine whether the MMN could be used as an objective measure of voice familiarity. More specifically, this study tempted to verify whether the evoked MMN response was of greater amplitude when the deviant stimulus is a familiar voice as opposed to an unfamiliar voice, as it may suggest that pre-attentive mechanisms are implicated in voice recognition. The main result of the present study is the significant difference between the MMN area under the curve elicited by a familiar voice when compared to that of an unfamiliar voice (Fig. 4). 
Figure 4. Grand-average MMNs elicited by a familiar voice (dashed) and by an unfamiliar voice (dotted), referenced to the standard stimulus ERPs (black).

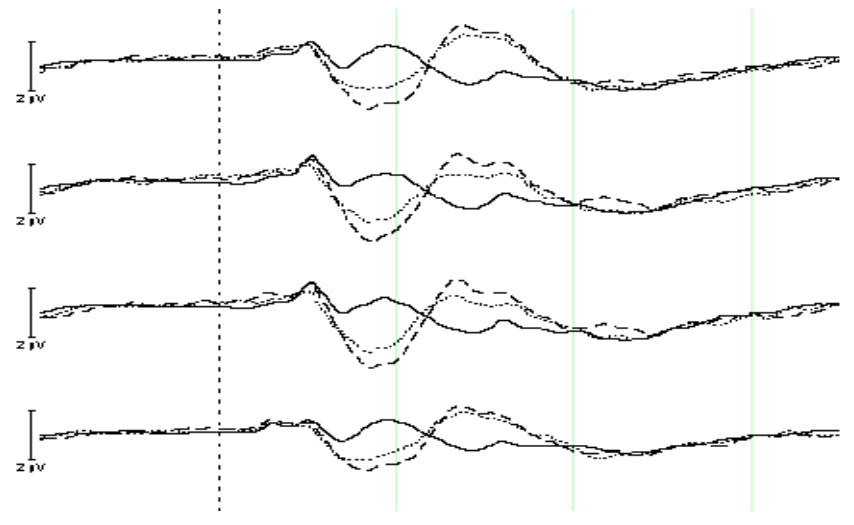

The dilemma

Before considering looking at variables of interest (amplitude, latency, area under the curve) stated in section 2, ERP data must first be filtered and analysed using a computer program such as BrainVision Analyser (refer to the Vision Analyser User Manual version 1.05 (C) Brain Products GmbH 1999 - 2004 for more detailed information). This program enables users to extract the MMN component waveform by scripting a macro (a specific program) specifically designed to subtract ERPs evoked by a standard stimulus from ERPs evoked by the presentation of a deviant stimulus (to access the step-by-step method of calculation of the $\mathrm{MMN}$, refer to Annex I). Once the MMN waves are obtained, we can then consider examining the variables of interest, as statistical analysis requires optimized quantification of the MMN.

The latency of the MMN can be interpreted as the time required to distinguish a deviant stimulus from a standard stimulus. In terms of sensory discriminations, the difference in timing when processing different stimuli is thought to account for the discrimination of subtle differences between the presented stimuli. When interpreting the latency of the MMN, it is important to disentangle the level of difficulty of the discrimination task from the timing of the discrimination process. In fact, if one wishes to determine which type of discrimination occurs earlier in the auditory system, it is essential to control for discrimination task difficulty when measuring $\mathrm{MMN}$ latencies. Applied to the above example, when comparing MMN elicited by a familiar voice to that of an unfamiliar voice, if the latencies of the two MMN components were found to be different, it would suggest that one voice is analysed prior to the other. As illustrated in Figure 4, no latency differences were found between the MMN elicited by a familiar voice with that elicited by an unfamiliar voice. Thus, latency computations will not be the focus of this paper as a fairly straightforward set of operations is sufficient to obtain this variable using the BrainVision Analyser program (refer to the Vision Analyser User Manual version 1.05 (C) Brain Products GmbH 1999 2004).

Another variable that has traditionally been extracted when analysing the MMN component is its amplitude, using averages over various time intervals, such as an interval around the peak latency. The amplitude of the MMN generally increases as the difference between the standard and the deviant stimuli is enhanced. This relationship is generally monotonic although it tends to level off as the difference between the standard and the deviant stimuli becomes large (Schröger \& Winkler, 1995). Therefore, the amplitude should not be utilized to quantify the MMN. Other investigators seem to prefer reporting the area under the curve to account for the size of MMN activation (Pekkonen et al., 1993; Sharma et al., 1993; McGee et al. 1997). However, the duration of the temporal window in which the MMN waveform occurs varied fairly across participants (Figures 5 and 6). Should one consider the peak amplitude to be the most indicative variable to reflect brain activation or is the area under the curve contained within a predefined temporal window more appropriate? How can experimenters account for such variability in the MMN waveform configuration?

Figure 5. MMN elicited by the same deviant stimulus for two different pariticipants at the same electrode site. Note that although both have the same amplitude, one is wider than the other one.

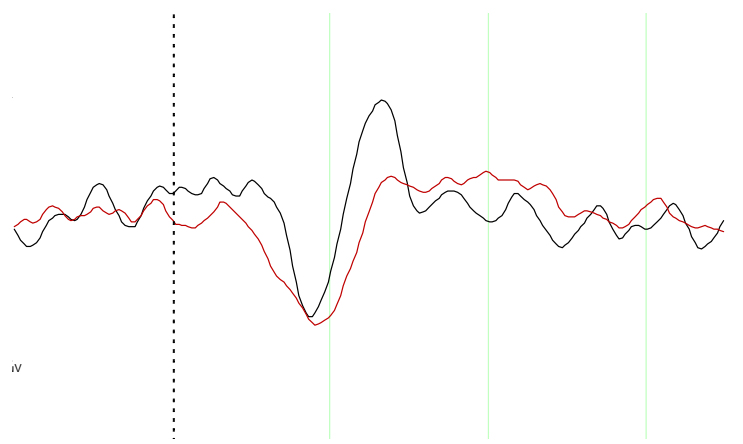

Figure 6. MMN elicited by the same deviant stimulus for two different participants at the same electrode site. Note here that although both have the same width, their amplitudes differ.

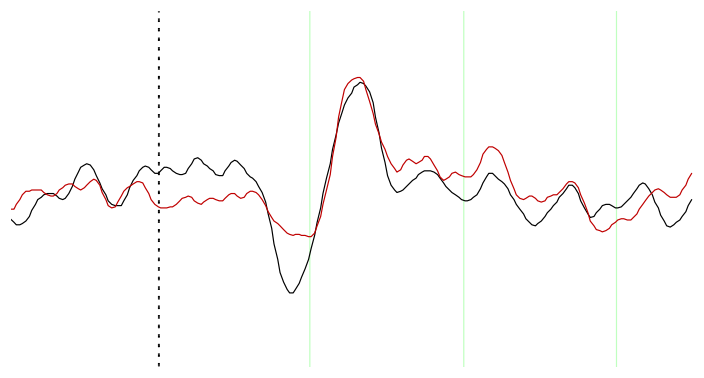




\section{A solution}

This section will attempt to describe what we consider to be the most appropriate way to resolve the high variability in MMN waveform configuration to account for brain activation. Thus, in order to develop an objective way to quantify the curve of a MMN component, advantages from both approaches (amplitude and area under the curve) were used.

Therefore, as used in the applied example, the MMN component was obtained using the area under the curve contained within a $50 \mathrm{~ms}$ time window in which the midpoint had previously been identified in a peak amplitude detection manipulation performed for each participant. The MMN component values obtained when presented with an unfamiliar voice were then compared to those elicited by a familiar voice. This method appears to be optimal since using a predefined window in which to compute the area under the curve prevents investigators to insert potential bias by the variability of the duration of the temporal window in which the MMN waveform occurs. Moreover, centering this defined window around the peak amplitude accounts for the greatest difference between the standard and deviant stimuli. Appendix II provides a detailed description on how to use BrainVision Analyser to obtain the area under the curve values.

\section{Conclusion}

Although the MMN has been useful in furthering scientific knowledge about auditory processing, its use is considerably limited by the interindividual response variability. Nevertheless, studies are systematically addressing the issue of test-retest reliability (Escera \& Grau, 1996; Tervaniemi et al., 1999; Pekkonen et al., 1995), while other groups are actively looking at ways to enhance the objective quantification of the elicited MMN response (Ponton et al., 1997; McGee et al. 1997). The present paper also intended to propose a new perspective on MMN analysis. Despite recent methodological advances that enabled investigators to reduce interindividual variability, it remains unclear whether the $\mathrm{MMN}$ is sufficiently reliable to be used in clinical settings. However, the MMN is one of the few biological indexes of fine-tuned sensory perception as it will most likely continue to yield significant insights about the processing of auditory information in various research and clinical endeavours.

\section{References}

Beauchemin, M., De Beaumont, L., Turcotte, A., Arcand, C., Vannasing, P., \& Belin, P., Lassonde, M. (submitted). $\mathrm{MMN}$ : an electrophysiological marker of voice familiarity. European Journal of Neuroscience.
Böttcher-Gandor, C., \& Ullsperger, P. (1992). Mismatch negativity in event-related potentials to auditory stimuli as a function of varying interstimulus interval. Psychophysiology, 29, 546-550.

Cowan, N., Winkler, I., Teder, W., \& Näätänen, R. (1993). Memory prerequisites of mismatch negativity in the auditory event-related potential (ERP). Journal of Experimental Psychology, Learning, Memory and Cognition, 19, 909-921.

Escera, C., \& Grau, C. (1996). Short-term replicability of the mismatch negativity. Electroencephalography and Clinical Neurophysiology, 100, 549-554.

Girard, M. H., Perrin, F., Pernier, J., \& Bouchet, P. (1990). Brain generators implicated in the processing of auditory stimulus deviance: A topographic event-related potential study. Psychophysiology, 27, 627-640.

McGee, T., Kraus, N., \& Nicol, T. (1997). Is it really a mismatch negativity? An assessment of methods for determining response validity in individual subjects. Electroencephalography and Clinical Neurophysiology, 104, 359-368.

Näätänen, R. (1995). The mismatch negativity: a powerful tool for cognitive neuroscience. Ear and Hearing, 16, 6-18.

Näätänen, R., \& Alho, K. (1995). Mismatch negativity - A unique measure of sensory processing in audition. International Journal of Neuroscience, 80, 317-337.

Näätänen, R., Gaillard, A. W. K., \& Mäntysalo, S. (1978). Early selective attention effect on evoked potential reinterpreted. Acta Psycologica, 42, 313-329.

Pekkone, E., Jousmäki, V., Partanen, J., \& Karhu, J. (1993). Mismatch negativity area and age-related auditory memory. Electroencephalography and Clinical Neurophysiology, 87, 321-325.

Pekkonen, E., Rinne, T., \& Näätänen, R. (1995). Variability and replicability of the mismatch negativity. Electroencephalography and Clinical Neurophysiology, 96, 546-554.

Ponton, C.W., Don, M., Eggermont, J. I., \& Kwong, B. (1997). Integrated mismatch negativity (MMNi): A noise free representation of evoked responses allowing single-point distribution-free statistical tests. Electroencephalography and Clinical Neurophysiology, 104, 381-382.

Schröger, E., \& Winkler, I. (1995). Presentation rate and magnitude of stimulus deviance effects on human preattentive change detection. Neuroscience Letters, 193, 185188.

Sharma, A., Kraus, N., McGee, T., Carrell, T., \& Nicol, T. (1993). Acoustic versus phonetic representation of speech as reflected by the mismatch negativity eventrelated potential. Electroencephalography and Clinical Neurophysiology, 88, 64-71.

Tervaniemi, M., Lehtokoski, A., Sinkkonen, J., Virtanen, J., 
Ilmoniemi, R. J., \& Näätänen, R. (1999). Test-retest reliability of mismatch negativity for duration, frequency, and intensity changes. Clinical Neurophysiology, 110, 1388-1393.

\section{Appendix I. Procedure to follow to obtain the MMN}

The procedure followed to extract the MMN component will be demonstrated using data from the applied example. Firstly, you must filter ERP data for artefacts and ocular corrections (refer to Vision Analyser User Manual (version 1.05 (C) Brain Products GmbH 1999 - 2004). Once these preliminary steps have been performed, the MMN component will be obtained using a pre-programmed macro, which requires an active history node for operation (make sure you selected the deviant stimulus node before activating the macro). This macro is programmed so as to subtract the ERP elicited by a frequent stimulus from that of a deviant stimulus.

Figure 7. Note that all the filtering and analysing steps are done. The VoixE $B$ is one of the two deviant stimuli. Il is selected so as to process the macro.

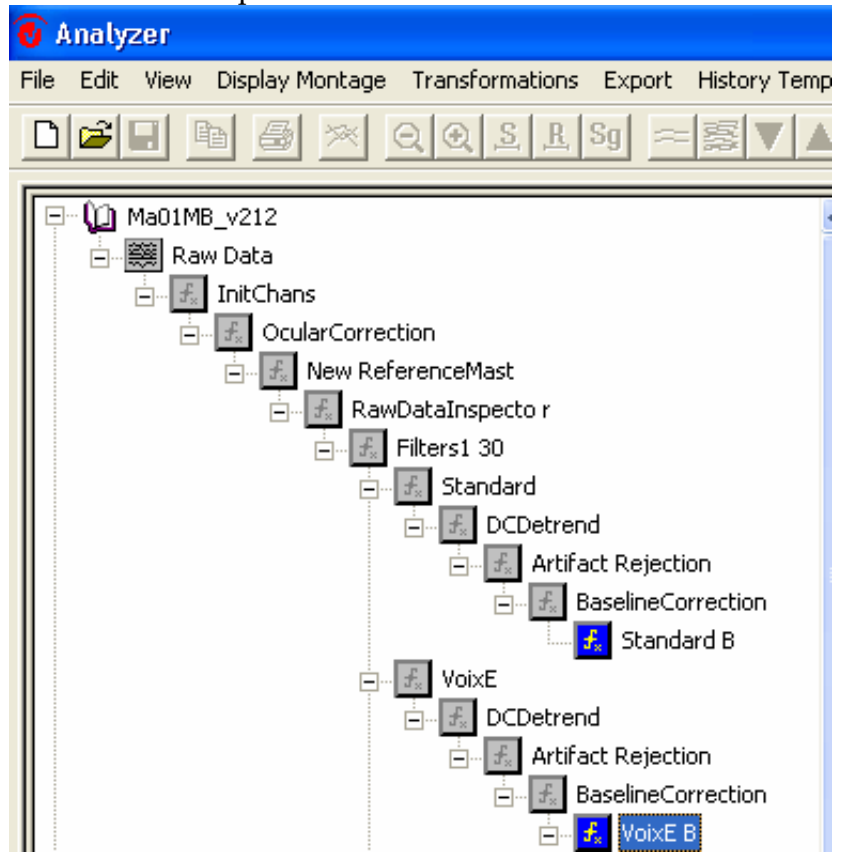

Then you click on Macro > Run to obtain the Run Macro dialog box. Select the CompareNodes program, which will allow you to perform the subtraction requested for the computation of the MMN. A second dialog box, specific to CompareNodes, will then appear. In this dialog box, you will need to specify the history nodes you intend to subtract from another.
Figure 8. The Run Macro dialog box.

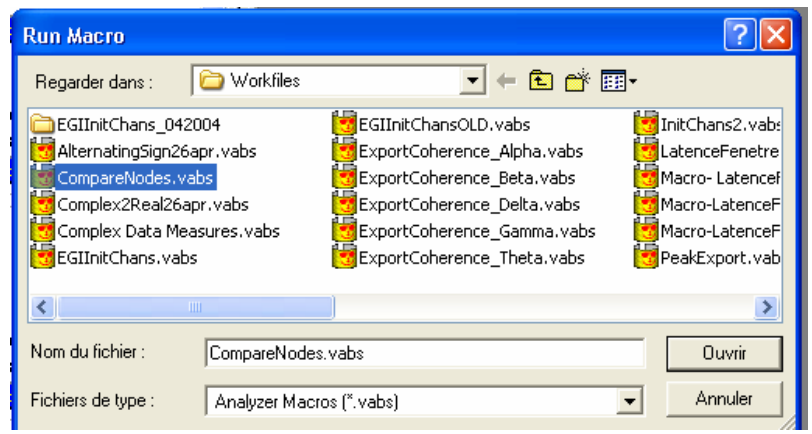

Figure 9. The CompareNodes dialog box.

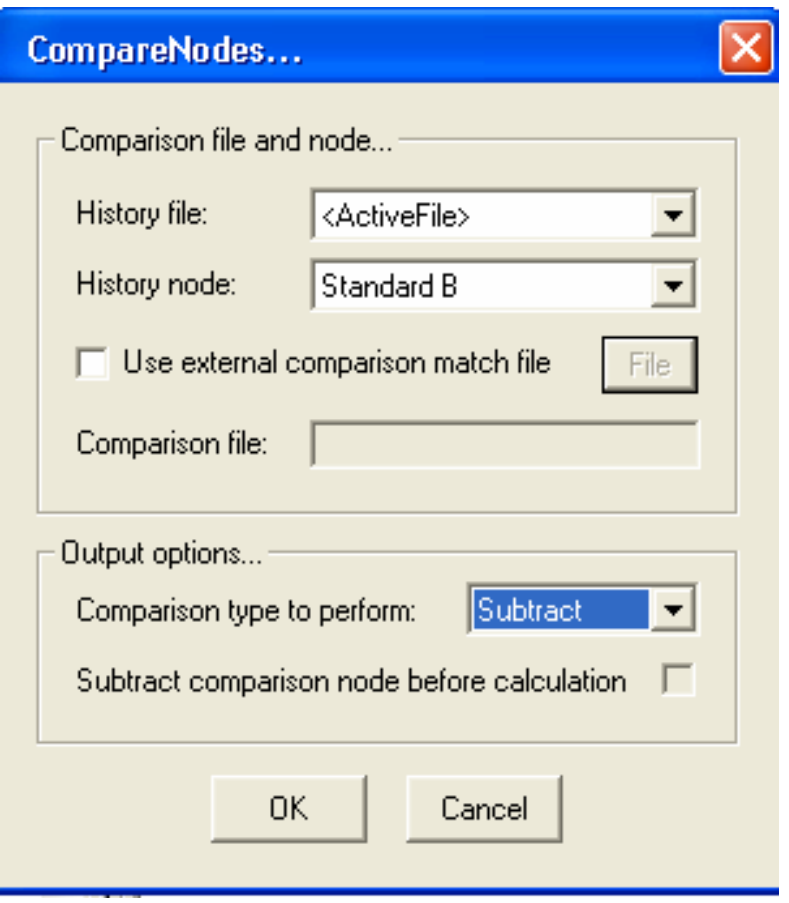

The History file field defines the node from which subtraction will be performed. Make sure to keep the default ActiveFile option as it reflects the previously selected deviant stimulus node. The History node field contains what will be subtracted from the Active File. To select the appropriate History node, use the arrow and scroll down until you find the desired standard stimulus. In the Comparison type to perform field, of the Output options section, select Subtract and then press OK. 
Figure 10. The history tree showing the added node for the MMN of one deviant stimulus.

\section{Analyzer - [Raw Data/InitChans/OcularCorrection/New Refere}

$\square$ File Edit Yiew Display Montage Transformations Export History Temp

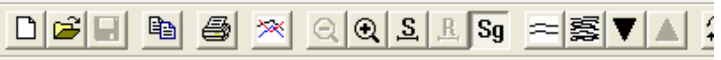

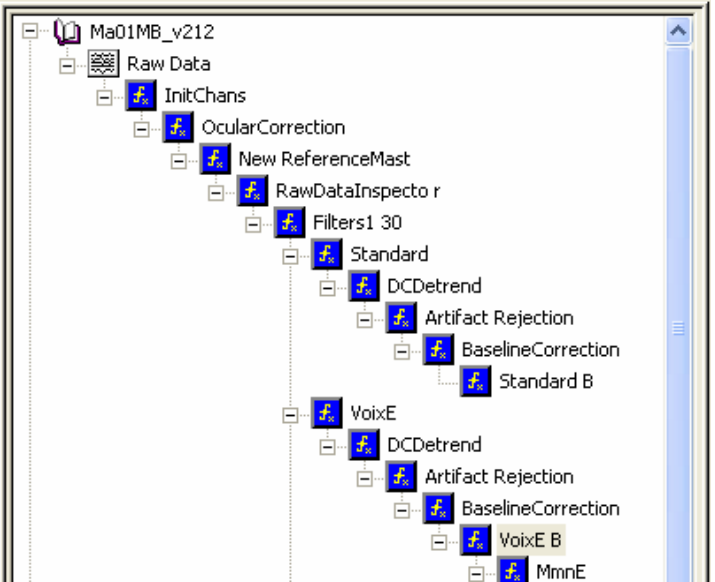

Note that an extra node that contains your extracted MMN was added. Repeat the above steps to obtain the MMN for the other deviant stimulus of interest.

\section{Appendix II. The new innovative area under the curve}

\section{variable}

Once you have completed the steps in Appendix I, a peak detection procedure is then executed to identify the most negative deflection in a literature-based predefined time window (between $80-280 \mathrm{~ms}$ ). In order to perform this peak detection step, you must first select the MMN node obtained after you have accomplished the Appendix I procedure of one deviant stimulus (in the applied example, either the familiar or the unfamiliar voice).

Figure 11. The MMN node obtained after you have accomplished the Appendix I is selected.

\section{Analyzer - [Raw Data/InitChans/OcularCorrection/New Referen} File Edit View DisplayMontage Transformations Export History Temp

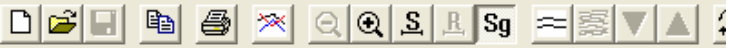

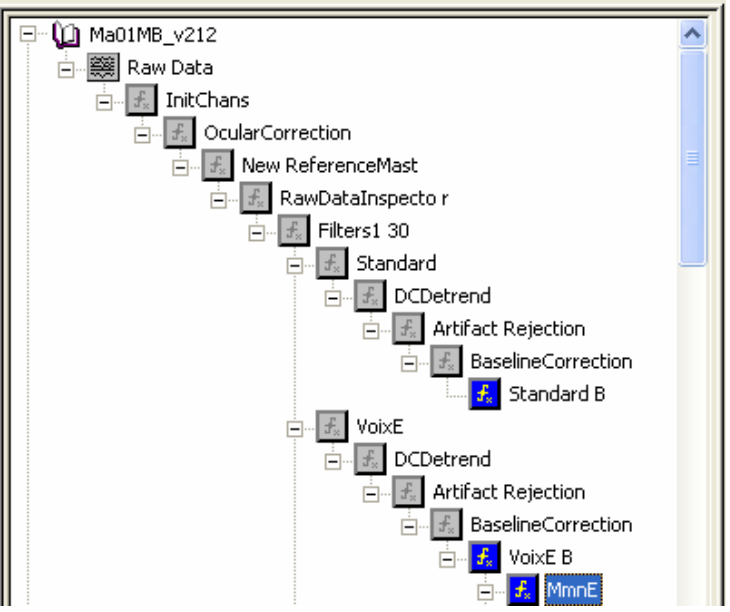

You click on Transformations $>$ Peak Detection to initiate a 3-step procedure for peak detection to occur.

Figure 12. Peak Detection dialog box - Step 1 of 3.

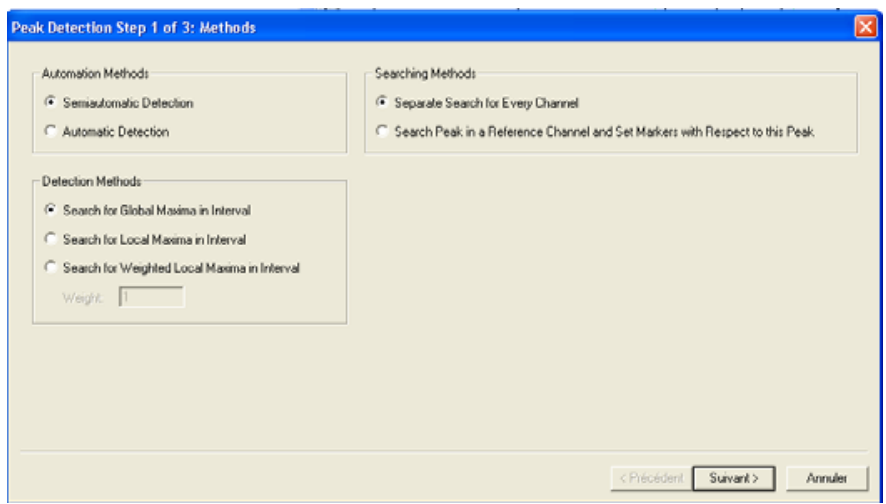

This firs step allows you to control for the degree of automation. With the semiautomatic detection, a cursor will be positioned where the algorithm detected the peak. In the Searching Methods section, you have to choose the option "Separate Search for every channel". You also have to choose the desired search method for peaks in the Detection Methods section. In searching for a global maxima, the edge points of the interval will be included when looking for peaks within the interval, rather than the local maxima detection method, which would exclude these edge points.

Figure 13. Peak Detection dialog box - Step 2 of 3.

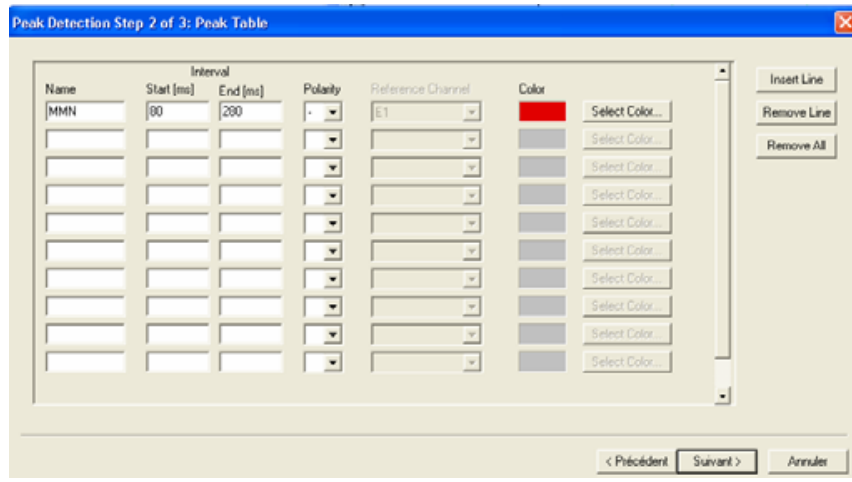

On the second page of the dialog box, you have to name the peak that you are searching for, indicate the window in which the peak must be searched in, as well as the polarity of the peak. On the third pane, you must enable channels of interest and click on Finish when this is done. Go through step one to three for the other deviant stimulus. 
Figure 14. Peak Detection dialog box - Step 3 of 3.

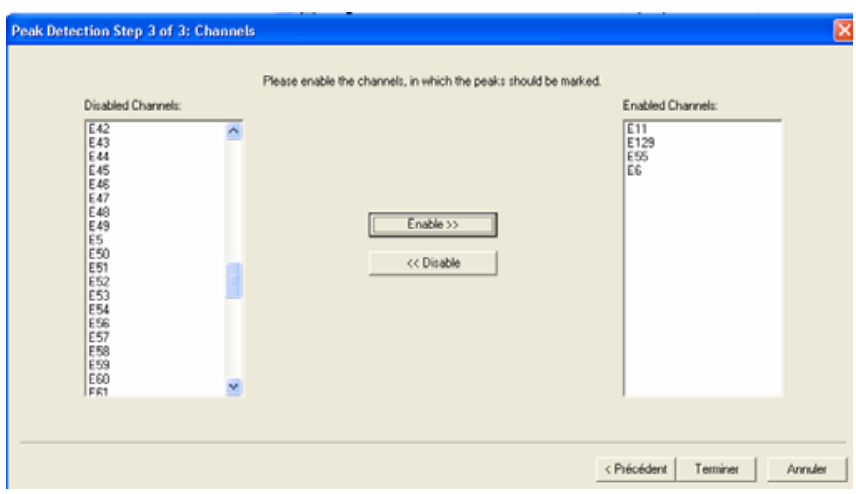

Now that the most negative point of the MMN has been peaked, you can now require BrainVision to calculate the area under the curve, as follows.

First select the Peak node of the MMN for one deviant stimulus. Then click on Export > Area Information Export.

Figure 15 The Peak node newly created is selected in order to perform the area under the curve calculation.

\section{Analyzer - [Raw Data/InitChans/OcularCorrection/New Referen $\square$ File Edit View DisplayMontage Transformations Export History Temp

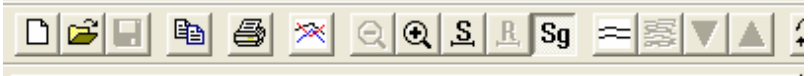

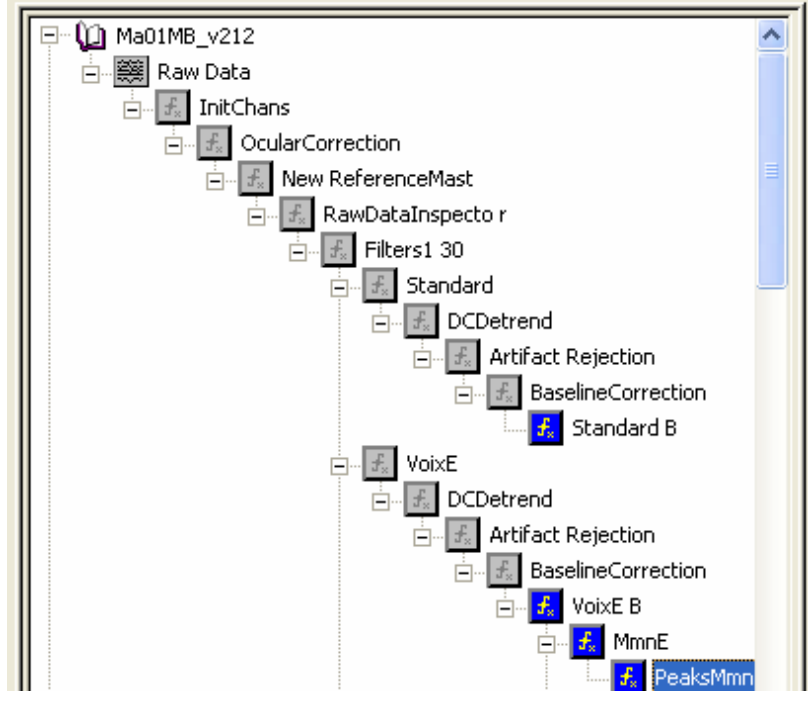

An Area Information Export dialog box will then appear. It allows you to export the area information of an interval. Specify in the Input section, that the calculation of the area under the curve is Time Domained. You then have to enter the values of the interval of interest (Area Interval Relative to Time 0 ), which you will obtain by subtracting and adding (by hand) $25 \mathrm{~ms}$ to the value found by the peak detection procedure that has just been performed. You also are requested to enter the Name of the Involved Data Sets, the peak node that you have selected just before requesting the Area Information Export window. By checking the Primary History Files Only, the selection will be confined to primary history files only. It is important to select the Individual History Files option as you do not want to include all files in the workspace. Remember that the interval in which the area under the curve will be measured must be calculated individually for each participant and each deviant stimulus peak detection. In the Available Files section, you will see all of your files, select the appropriate one by clicking the Add button. The selected file will then be transferred into the Selected Files section. Make sure to Use Activity to rectify the sign so the values are unsigned and to select the Area Export Type. Finally name your output in the Output File field of the Output section. You have to repeat all the above steps for each participant for both deviant stimuli. Once you have completed those steps, you can open the output files in a Microsoft Excel sheet. Organize you data so as to facilitate statistical analyses performed with SPSS or any other statistical program of your choice.

Figure 16. The Area Information Export dialog box.

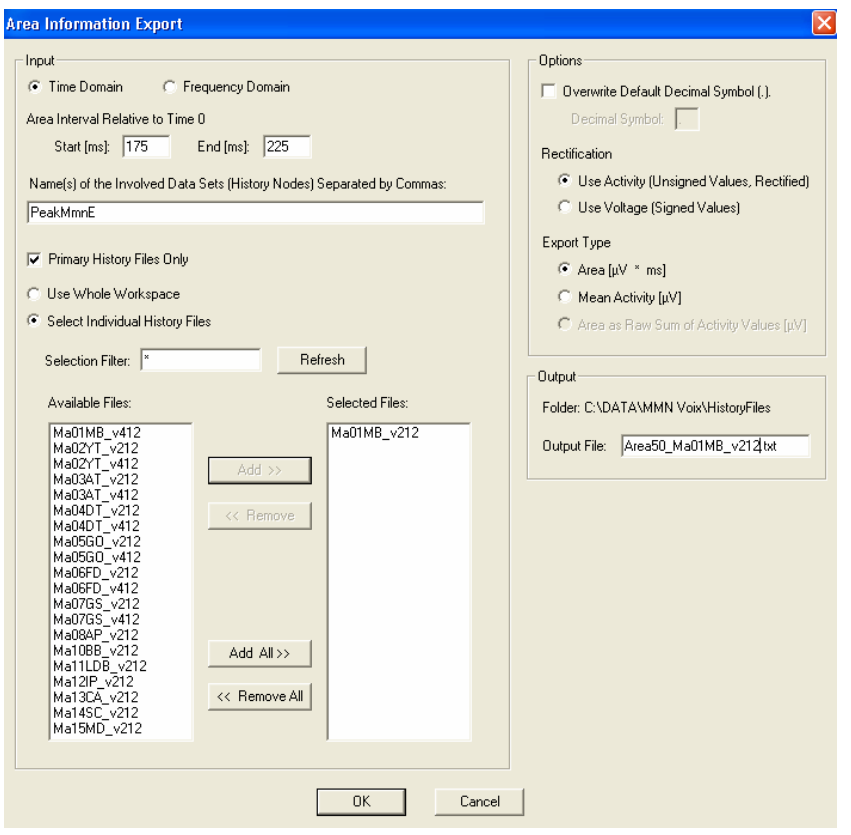

Received July 12, 2005

Accepted September 22, 2005 